\title{
Network formation with heterogeneous agents and absolute friction*
}

\author{
Joost Vandenbossche ${ }^{\dagger}$ and Thomas Demuynck ${ }^{\ddagger}$
}

August 30, 2010

\begin{abstract}
We present a model of strategic network formation with absolute friction and heterogeneous agents. The individual payoffs from a given network are determined by the difference of an agent specific utility function that depends on the number of his/her direct links and the sum of his/her link-costs. These link-costs decompose in a symmetric function that represents the social distance between the two agents and an agent specific function representing the partner's quality. From a theoretical point of view, we analyze strong pairwise stable networks (see Belleflamme and Bloch (2004) and show that our model always has a unique strong pairwise stable network which is also strongly stable (as in Dutta and Mutuswami (1997). From a practical point of view, we provide an algorithm that reproduces this stable network from information on the individual payoff structure. We illustrate the use of this algorithm by applying it to an informal risk sharing network data set from the village of Nyakatoke in rural Tanzania.
\end{abstract}

Keywords: network formation, heterogeneity, absolute friction

JEL-classification: C62; C78; C79

*We sincerely thank Dirk Van de gaer for his valuable recommendations and insightful comments on an earlier draft of this paper. We acknowledge financial support from the Fund for Scientific Research Flanders (FWO - Vlaanderen), the Interuniversity attraction poles programme - Belgian Science Policy [Contract $\mathrm{N}^{\circ} \mathrm{P} 06 / 07$ ] and FWO project $\mathrm{N}^{\circ}$ G.0078.05.. Part of this research was completed during a research visit at Cambridge university at the beginning of 2009.

${ }^{\dagger}$ Corresponding author: Sherppa, Ghent University, Tweekerkenstraat 2, 9000 Gent, Belgium. email: joost.vandenbossche@ugent.be

${ }_{\ddagger}$ Center for Economic Studies, Catholic University of Leuven, and Sherppa, Ghent University. email:thomas.demuynck@kuleuven-kortrijk.be 


\section{Introduction and motivation}

We present a model of strategic network formation with absolute friction and heterogeneity among agents. We show that our model always has a unique network that is strong pairwise stable. We provide an algorithm to compute this network and we illustrate its use by applying it to a data set of a risk sharing network in small village in Tanzania.

Overview Social networks are among the most valuable contributors to apprehend modern human life. They determine, among others, communication, friendship, trust, marriage, trade, risk sharing and wealth. In order to understand these phenomena it is crucial to study how these networks are formed and what a stable network looks like when agents are able to form and/or cut links. This insight has lead to an enormous growth of scientific research analyzing the development, stability and empirical regularities of social networks.

In this research we present a model of strategic network formation. Our model has three important features. First, the model assumes that link formation is only possible under mutual consent. This means that in order to establish a link, two agents have to agree to form this link, while every agent can unilaterally decide to cut a pre-existing link (see Jackson and Wolinsky (1996)). This feature distinguishes our research from models of unilateral link formation. ${ }^{1}$ In models of unilateral link formation, any agent can unilaterally decide to link with another agent. In this respect, it is also useful to mention that our model does not allow for transfers between agents, where it is possible that one agent compensates another agent for the formation a link between them. ${ }^{2}$ Second, our model allows for a wide range of heterogeneity in the determination of the link-costs. The link-cost is the cost that each agent incurs when adding a certain link. Third, we restrict ourselves to networks with absolute friction. This means that the benefits of a certain link only attribute to the two agents that form this link, excluding any spillover to other agents. In this section, we discuss each of these three features in more detail. This will allow us to position and distinguish our research from other research in the network formation literature.

Bilateral link formation We assume that links can only be formed under mutual consent. In particular, no agent can form a link with another agent without the agreement of this agent but any agent can unilaterally decide to cut an existing link (see ?Myerson, 1991; Jackson and Wolinsky, 1996). We say that a network is stable if there is no coalition of agents that can alter the network in such a way that at least one agent in the coalition benefits from the change without any agent in the coalition losing from the change. ${ }^{3}$ This definition hinges on two further specifications. First, we need to specify which coalitions

\footnotetext{
${ }^{1}$ See, among others, ?Dutta and Jackson (2000); Bala and Goyal (2000); ?); Haller and Sarangi (2005); Galeotti, Goyal, and Kamphorst (2006); Feri (2007); ?); ?.

${ }^{2}$ See, among others, Dutta and Mutuswami (1997); Dutta, Tijs, and van den Nouweland (1998); Slikker and van den Nouweland $(2000,2001) ; ?) ; ?$.

${ }^{3}$ This definition abstains from various forms of farsightedness (see for example Watts (2002); Deroian (2003); Watts (2002); Dutta, Ghosal, and Ray (2005); ?); ?); ? or evolutionary dynamics (see, for instance, ?Jackson and Watts (2002); Dutta et al. (2005); ?); Carayol and Roux (2006); Feri (2007); ?).
} 
are allowed to alter the existing network, and second, we need to specify in which manner these coalitions may alter the current network.

Depending on the specifications of coalition size and alterations, different stability concepts can be established. At the one extreme we may allow for coalitions of arbitrary size that can modify a given network by creating any link between two agents within the coalition and cut any number of links that involves at least one agent from the coalition. This corresponds to the notion of strong stability as introduced by Dutta and Mutuswami (1997) and Jackson and van den Nouweland (2005). At the other end of the spectrum we may consider the pairwise stability concept where deviating coalitions have size at most two and these coalitions can change the network by either creating the link between the two agents in the coalition or either cut a single link involving an agent in this coalition (see, Jackson and Wolinsky (1996); Jackson and Watts (2001)). An intermediary stability concept which we will discuss, is the concept of strong pairwise stability, introduced by Belleflamme and Bloch (2004). In this case, the maximal coalition size is two, as for the pairwise stability concept, but all types of alterations available to the agents in the coalition are allowed, as for the strong stability concept. Hence, a pair of agents may cut as many links as they like and simultaneously form a link between them. We show that our model always has a unique strongly (pairwise) stable network.

Heterogeneity in the payoff structure Within this model of network formation under mutual consent, we define the payoff structure for the various agents. The payoff from being part of a given network consists of a benefit part and a link-cost part, reflecting the idea that agents have to incur costs to establish a link. Agents have to invest time and effort to build a relationship and agree on the conditions of the link. We assume that this link-cost can be split into two parts. The first part is a pairwise component which we will call distance $d$ and is symmetric - i.e. for all agents $i, j$ in a population $N$ the distance from $i$ to $j$ is equal to the distance from $j$ to $i$. Distance reflects the idea that the better the exante relationship between agents is, the less time and effort needs to be invested, because of a pre-established level of trust and understanding or better monitoring possibilities. The second part is a agent-specific, idiosyncratic, component which we will call quality $\theta$. The assumption is made that increased quality of the linking partner reduces link-costs. ${ }^{4}$

All other studies of network models with bilateral link formation use more restrictive forms for the cost topology. Among others, Jackson and Wolinsky (1996); ?); ? analyze models with homogeneous costs, i.e. the setting where linking costs are constant for all pairs of agents. Johnson and Gilles (2000) assume that costs are described by a line cost topology. In this model, agents are situated on a line and the linking-cost between two agents is determined by the Euclidean distance between them. Carayol and Roux (2006) uses a circle-cost topology, where agents are situated on a circle and the linkingcost between two agents is determined by their shortest distance. Finally, Jackson and Rogers (2005) impose an island cost topology. In this setting, agents are allocated to a

\footnotetext{
${ }^{4}$ This impact may be indirect in that a higher quality may be reflected in an extra benefit, which increases the willingness to link, effectively reducing link-cost in an additive framework.
} 
finite number of islands and the linking-cost between two agents may take on one of two values conditional on the requirement that the link-cost between two agents on the same island is less costly than the link-cost between agents on distinct islands.

More general models of heterogeneity were investigated for models with non-bilateral link formation. In particular, Galeotti, Goyal, and Kamphorst (2006) investigate a model of one sided-link formation where costs can vary freely. Finally, ? and Haller and Sarangi (2005) use the same heterogeneity concepts as we do - distance and quality - in a network formation model with probabilistic graphs. In probabilistic graph models, link formation may fail with a certain probability(?). More specifically, ? assumes that this probability depends on the effort - costs - made by the agents involved. Hence, a major contribution of our model is that it is the first model which combines bilateral link formation with such general forms of heterogeneity.

Absolute friction In addition, and contrary to previously mentioned studies of bilateral link formation, our model assumes absolute friction. Absolute friction states that the benefits from the network to a certain agent only depend on the agents that are directly linked to this agent. A model with less than absolute friction assumes that the benefits are decreasing in the length of the path while a model with no friction assumes that the length of the path does not matter in defining the benefits. The assumption of absolute friction is necessary to keep our model tractable. Indeed, the restrictive nature of the cost heterogeneity used in the literature with models of bilateral link formation formation (see above) strongly indicates that models with moderate levels of friction are only tractable by imposing more stringent restrictions on the cost topology.

The assumption of absolute friction is defendable for networks of informal insurance, trust or trade. Fafchamps and Lund (2003) show that risk sharing is not frictionless due to transaction costs, imperfect commitment, asymmetric information or other processes that limit exchange. This finding is corroborated by De Weerdt and Dercon (2006) who indicate that direct network partners may be most relevant for understanding risk sharing. In addition, we could also assume that agents are shortsighted because they do not know the complete structure of the network and only take into account the benefits from their direct partners, while assuming other benefits to be non-existent. In this case, the network that emerges looks like the network that would emerge in the case of absolute friction, so that the absolute friction assumption is valid in this setting.

When we do not assume shortsightedness the assumption of absolute friction may be more difficult to maintain. In certain settings, like communications networks, an agent with many connections may be appealing to link to because she may pass on to you the information she got from her connections. Likewise, in a collaboration network an agent with many connections may be less attractive since his time is taken by many agents, leaving less time to work together. ${ }^{5}$ In these cases, the indirect network matters. Thus, we

\footnotetext{
${ }^{5}$ See, among others, the connections and co-author models in Jackson and Wolinsky (1996), the job contact network in ? and the local spillover game in ?.
} 
conclude that the validity of our assumption of absolute friction depends on the context and the additional assumptions made.

Main results This paper contributes to the present literature by providing a tractable model of two-sided network formation with few restrictions on linking costs, while considering a wide range of stability concepts. We show that for our model, there always exists a unique stable network which is both strongly and strong pairwise stable.

Besides the existence and uniqueness result, we provide an algorithm that reproduces this stable network. In this algorithm, starting from the complete network, we let a sequence of agents cut the links they do not want to maintain, given the cutting actions of the agents before them in the sequence. The stability of the outcome of the algorithm hinges on the order of the agents in the sequence. We can show that for the resulting network, in every iteration of the algorithm there will always be at least one agent whose set of links she would like to maintain in that network will be equal to her set of links in the unique stable network. Hence, the sequence of agents is such that in every iteration exactly those agents are selected. Naturally, an agent cannot be selected twice and is deleted from the selection pool once selected. This algorithm is one of the key innovations in this paper. Once we know the structure of the cost functions, and the form for the benefit functions, we can compute the particular sequence of agents and the emerging stable network, observe its general features and relate these features to these structures.

We illustrate the use of our algorithm by applying it to a data set on an informal risk sharing network drawn from the village of Nyakatoke in rural Tanzania. ${ }^{6}$ Although our approach is rather crude in the measurement of the cost structure, implying that our exercise can be considered as an illustration at best, we find that our algorithm outperforms a random network formation model where probabilities were fitted from a logit regression which uses the same data.

Section 2 introduces the model and provides the definition of a stable network. Section 3 contains and discusses the main result relating to the uniqueness and existence of the stable network. Section 4 provides the empirical illustration and section 5 concludes.

\section{The model}

Models of network formation Consider a set of agents $N=\{1, \ldots, n\}$. An undirected network $g$ is given by a collection of two-element sets $\{i, j\}$ where $i, j \in N$ and $i \neq j$. For ease of notation, we will write $i j$ instead of $\{i, j\}$. If $i j \in g$, we say that agents $i$ and $j$ are linked in the network $g$. We denote the complete network - the network where every agent is linked to every other agent- by $g_{N}$, i.e. $g_{N}=\{i j \mid i, j \in N, i \neq j\}$. We denote by $\mathcal{G}_{n}$ the collection of all networks on a set of $n$ agents. To each agent $i \in N$, we endow a payoff function $\pi_{i}$ from the set $\mathcal{G}_{n}$ to the set $\mathbb{R}$ defining the payoff, $\pi_{i}(g)$ that agent $i$

\footnotetext{
${ }^{6}$ This data set is also used in the research of Comola (2008); De Weerdt (2002); De Weerdt and Dercon (2006); ?.
} 
receives when the network $g$ is established. A payoff structure $\left\{\pi_{i}\right\}_{i \leq n}$ consists of a finite number, $n$, of agents and payoff functions $\pi_{i}$ for every agent $i \leq n$. We denote by $\mathcal{S}$ the set of all payoff structures.

Stability concepts A stability concept, $\Pi$, is a correspondence from the set of payoff structures, $\mathcal{S}$, to the set of all networks $\bigcup_{n} \mathcal{G}_{n}$ such that for all payoff structures, $\left\{\pi_{i}\right\}_{i \leq n} \in$ $\mathcal{S}$

$$
\Pi\left(\left\{\pi_{i}\right\}_{i \leq n}\right) \subseteq \mathcal{G}_{n}
$$

The set $\Pi\left(\left\{\pi_{i}\right\}_{i \leq n}\right)$ determines the set of stable networks corresponding to the payoff structure $\left\{\pi_{i}\right\}_{i \leq n}$ and stability concept $\Pi$.

We begin by defining two particular stability concepts. In order to model these concepts, we borrow the idea of a linking game as introduced by Myerson (1991). For the moment let us fix a payoff structure $\left\{\pi_{i}\right\}_{i \leq n}$ and the set of agents $N=\{1, \ldots, n\}$. For an agent, $i \in N$, his/her strategy set $S_{i}$ consists of all subsets of $N-\{i\}$. This definition includes the empty set, $\emptyset$. The interpretation is that $j \in s_{i}$ if $i$ proposes to $j$ to form a link and $j \notin s_{i}$ if $i$ is not willing to link with $j$. For a given strategy profile, $\left(s_{1}, \ldots, s_{n}\right)$, a link between $i$ and $j$ will be formed if $j \in s_{i}$ and $i \in s_{j}$. In other words, $i$ and $j$ will be linked if $i$ proposes to form a link to $j$ and $j$ proposes to form a link to $i$. We denote by $g\left(s_{1}, \ldots, s_{n}\right)$ the network that is formed when agent $i \in N$ chooses strategy $s_{i} \in S_{i}$. In particular, we will have that $i j \in g\left(s_{1}, \ldots, s_{n}\right)$ if and only if $j \in s_{i}$ and $i \in s_{j}$. Observe that, in general, there are several strategy profiles that may lead to the same network, i.e. there will exist distinct profiles $\left(s_{1}, \ldots, s_{n}\right)$ and $\left(s_{1}^{\prime}, \ldots, s_{n}^{\prime}\right)$ such that $g\left(s_{1}, \ldots, s_{n}\right)=g\left(s_{1}^{\prime}, \ldots, s_{n}^{\prime}\right)$. One notable exception is the complete network, $g_{N}$, where $g\left(s_{1}, \ldots, s_{n}\right)=g_{N}$ if and only if $s_{i}=N-\{i\}$ for all $i \in N$. For every coalition $C \subseteq N$ and strategy profile $s \in S$, we also write $s=\left(s_{C}, s_{N-C}\right)$, where $s_{C}$ is the strategy profile $s$ restricted to agents within the coalition $C$. We call $s_{C}$ a coalition strategy profile.

The first stability concept is strong stability as developed in Dutta and Mutuswami (1997) and Jackson and van den Nouweland (2005). A network is strongly stable if for all possible coalitions $C \subseteq N$ of agents there does not exist a deviation of the strategy profile by members of $C$ such that at least one agent within the coalition strictly gains from the deviation, and no agent within the coalition loses from this rearrangement. Formally, ${ }^{7}$

Definition 1 (Strong Stability). A network $g$ is strongly stable if there exists a strategy profile $\left(s_{1}^{*}, \ldots, s_{n}^{*}\right)$ such that $g=g\left(s_{1}^{*}, \ldots, s_{n}^{*}\right)$ and for all nonempty coalitions $C$, there does not exist a coalition strategy profile $s_{C}$ such that for all $i \in C, s_{i} \in S_{i}$ and

$$
\pi_{i}\left(g\left(s_{C}, s_{N-C}^{*}\right) \geq \pi_{i}\left(g\left(s_{C}^{*}, s_{N-C}^{*}\right)\right),\right.
$$

with at least one inequality being strict.

\footnotetext{
${ }^{7}$ We follow the definition as stated in Jackson and van den Nouweland (2005), since this definition is compatible with pairwise stability.
} 
For a given payoff structure $\left\{\pi_{i}\right\}_{i \leq n}$, we denote by $\Pi_{s s}\left(\left\{\pi_{i}\right\}_{i \leq n}\right)$ the (possibly empty) collection of strongly stable networks.

For our purposes, we also consider a second stability concept, strong pairwise stability as defined in Belleflamme and Bloch (2004), which restricts the size of deviating coalitions to at most two.

Definition 2 (Strong Pairwise Stability). A network $g$ is strongly pairwise stable if there exists a strategy profile $\left(s_{1}^{*}, \ldots, s_{n}^{*}\right)$ such that $g=g\left(s_{1}^{*}, \ldots, s_{n}^{*}\right)$ and for all nonempty coalitions $C$ of size less than or equal to 2 , there does not exist a coalition strategy profile $s_{C}$ such that for all $i \in C, s_{i} \in S_{i}$ and

$$
\pi_{i}\left(g\left(s_{C}, s_{N-C}^{*}\right)\right) \geq \pi_{i}\left(g\left(s_{C}^{*}, s_{N-C}^{*}\right)\right),
$$

with at least one inequality being strict.

For a given payoff structure $\left\{\pi_{i}\right\}_{i \leq n}$, we denote by $\Pi_{s p s}\left(\left\{\pi_{i}\right\}_{i \leq n}\right)$ the (possibly empty) collection of strongly pairwise stable networks. In the following section we will show that our model allows for a unique strong pairwise stable network that is also strongly stable.

For completeness we also give the definition of pairwise stability and we denote for a given payoff structure $\left\{\pi_{i}\right\}_{i \leq n}$, the collection of pairwise stable networks by $\Pi_{p s}\left(\left\{\pi_{i}\right\}_{i \leq n}\right)$.

Definition 3 (Pairwise stability). A network $g$ with strategy profile $s$ is pairwise stable if

- for all $i, j \in N$ such that $j \in s_{i}$ and $i \in s_{j}$ we have that $\pi_{i}\left(g\left(s_{i} / j, s_{-i}\right)\right) \leq \pi_{i}(g(s))$ and $\pi_{j}\left(g\left(s_{j} / i, s_{-j}\right)\right) \leq \pi_{j}(g(s))$.

- for all $i, j \in N$ such that $j \notin s_{i}$ or $i \notin s_{j}$ we have that if $\pi_{i}(g \cup\{i j\})>\pi_{i}(g)$ then $\pi_{j}(g \cup\{i j\})<\pi_{j}(g)$.

The main difference between strong pairwise stability and pairwise stability lies in the fact that the former allows for simultaneous cutting and forming of a link while the latter does not.

Model specification We are now ready to define a specific functional structure of the payoff functions $\pi_{i}$. We assume that the payoff for $i$ of a network $g, \pi_{i}(g)$, can be decomposed in two parts. A first part provides the benefit that $i$ obtains from the network $g$ and a second part provides the costs that $i$ incurs from the formation of the network $g$.

For the benefit part, we impose the assumption of absolute friction, meaning that agents do not have any advantage or disadvantage of the presence of indirectly connected agents. In particular, each agent $i \in N$ is endowed with an agent specific utility function $v_{i}: \mathbb{N} \rightarrow \mathbb{R}$ representing the utility that $i$ derives from her number of direct links $n_{i}(g)$,

$$
n_{i}(g)=\left|L_{i}(g)\right| \equiv|\{j \in N-\{i\} \mid i j \in g\}| .
$$

By making the assumption that only the number of direct links matter, we impose homogeneity on the benefit side, i.e. all potential partners are deemed to be equal in terms 
of linking gains. Furthermore, we assume that the change in utility is strictly decreasing in the number of links and that the additional benefit of adding an extra link becomes arbitrarily small or negative if the number of links is large enough. Formally, for all $l \in \mathbb{N}$ :

a) $v_{i}(l+2)-v_{i}(l+1)<v_{i}(l+1)-v_{i}(l)$ and,

b) for all $\varepsilon>0$, there exists a number $l(\varepsilon) \in \mathbb{N}$ such that for all $l \geq l(\varepsilon), v_{i}(l+1)-v_{i}(l) \leq$ $\varepsilon$.

Establishing and maintaining a link is costly. We introduce the notion of a link-cost function to model these linking costs. As mentioned in the introduction, we assume that this link-cost function depends on two factors: a distance factor and a quality factor. We can think of social networks where, if the social distance between agents is large, they will have to incur more costs to overcome this distance and establish a link. For every pair of agents $i$ and $j$, we define a number $d(i, j)$ representing the distance between $i$ and $j$. We assume that this function is symmetric. Formally,

c) for all $i, j \in N: d(i, j)=d(j, i)$.

Besides this distance function, we assume that the cost of link formation is also determined by a component idiosyncratic of the partner agent which we call the quality of the agent. For each agent we consider a number $\theta(i)$ that represents this quality. The larger $\theta(i)$ the higher the quality of agent $i$. For $i$ to link to $j$, the quality of agent $j, \theta(j)$, has a decreasing impact on link-costs. Hence, we assume that the cost of $i$ from linking with $j$, $c(i, j)$, can be written as a difference between these two parts:

$$
c(i, j)=d(i, j)-\theta(j)
$$

At the end of section 3, we show that our model can be adapted to include alternative forms for this cost function.

Finally, in order to obtain our uniqueness result, we impose two additional assumptions:

d) for all $i, j$ and $k \in N: c(i, j) \neq c(i, k)$ and,

e) for all $l \in \mathbb{N}$ and all $i$ and $j \in N, v(l+1)-v(l) \neq c(i, j)$.

The first assumption states that every agent can perfectly order the other agents in terms of linking costs, without there being any indifference between potential linking partners. The second assumption implies that no agent can ever be indifferent between having a certain link or not. These requirements are generic in the sense that any violation of them will be undone for a small perturbation in the distance or quality functions. No other assumptions are made with respect to the cost structure. Note that this makes it possible that $c(i, j)<0$. 
Bringing together the cost and benefit sides, we get that the payoff of $i$ derived from the network $g$ is given by,

$$
\begin{aligned}
\pi_{i}(g) & =v_{i}\left(n_{i}(g)\right)-\sum_{j \in L_{i}(g)} c(i, j) \\
& =v_{i}\left(n_{i}(g)\right)-\sum_{j \in L_{i}(g)}(d(i, j)-\theta(j)) .
\end{aligned}
$$

\section{Stable networks}

Having outlined the various concepts of stability and the specific payoff structure of our model, we proceed by showing existence and uniqueness of the strong pairwise stable network. We also give some additional insights into the configuration of this stable network. In particular, we provide an algorithm that allows the computation of the stable network from information on the individual benefit functions $v_{i}($.$) and cost structure c(i,$.$) . In$ this section, we restrict ourselves to providing the necessary definitions and concepts to understand our main results and the main idea behind the algorithm. We illustrate this algorithm by an example. The formal proof of our result can be found in the appendix.

Consider four agents, $N=\{1,2,3,4\}$. The distance, quality and cost functions are given below in table 1 together with the benefit function $v_{i}$ which is assumed to be identical for all $i=x_{1}, \ldots, x_{4}$. Observe that the cost function, $c(i, j)$, is constructed using the formula,

\begin{tabular}{|c|c|c|c|c|c|c|c|c|c|c|c|c|c|}
\hline \multicolumn{5}{|c|}{$d(i, j)$} & \multicolumn{2}{|c|}{$\theta(i)$} & \multicolumn{5}{|c|}{$c(i, j)$} & \multicolumn{2}{|c|}{$v(n)$} \\
\hline$i \backslash j$ & 1 & 2 & 3 & 4 & $i$ & & $i \backslash j$ & 1 & 2 & 3 & 4 & $n$ & \\
\hline 1 & 1 & 49 & 76 & 23 & 1 & 10 & 1 & t & 44 & 56 & 8 & 0 & 0 \\
\hline 2 & 49 & / & 58 & 16 & 2 & 5 & 2 & 39 & / & 38 & 1 & 1 & 100 \\
\hline 3 & 76 & 58 & / & 62 & 3 & 20 & 3 & 66 & 53 & / & 47 & 2 & 150 \\
\hline 4 & 23 & 16 & 62 & I & 4 & 15 & 4 & 13 & 11 & 42 & / & 3 & 180 \\
\hline
\end{tabular}
$c(i, j)=d(i, j)-\theta(j)$.

Table 1: Overview

The algorithm, given in figure 1 , selects for each iteration $t=0,1,2, \ldots$ a sets of agents $A_{t} \subseteq N_{t}$ - with $N_{t}$ a subpopulation of $N$ - who are allowed to change their strategy. This change in strategy changes a given network $g_{t}$ to the network $g_{t+1}$. More specific, the allow the agents in $A_{t}$ to delete the links in network $g_{t}$ they do not want to maintain. We start with the complete network $g_{0}=g_{N}$ and the grand population $N_{0}=N$. The algorithm guarantees that for each iteration $t=1,2, \ldots, g_{t} \subseteq g_{t-1}, N_{t}=N_{t-1}-A_{t} \subseteq N_{t-1}$ and $N_{t} \neq N_{t-1}$. We end the algorithm when $N_{t}=\emptyset$, lets say at iteration $T$, which is guaranteed to happen in finite time.

We now discuss each step in the algorithm, for a certain iteration $t$, and we relate it to our numerical example above. 
Assume that we arrive at step $t$ of the algorithm with network $g_{t}$ and coalition $N_{t} \subseteq N$. In order to determine $A_{t}$ we take the following steps.

In a first step, we compute for every $i \in N_{t}$ and $j \in N$ for which $i j \in g_{t}$, the number of agents, $k$, in $N$ such that $i k \in g_{t}$ and $c(i, k) \leq c(i, j)$. We denote this number by $r\left(i, j, g_{t}\right)$.

Definition 4. For all $i, j \in N$ and $g \in \mathcal{G}_{n}$,

$$
r(i, j, g)=\mid\{k \in N \mid i k \in g \text { and } c(i, k) \leq c(i, j)\} \mid .
$$

Considering our numerical example, we have that, for example, $r\left(1,3, g_{0}\right)=3$ : there are three agents, namely 2, 3 and 4 , who are linked to 1 in $g_{0}$ and have linking-costs less than or equal to $c(1,3)=56$.

In a second step, we compute for each $i \in N_{t}$ and $j \in N$ with $i j \in g_{t}$ the maximal number $t(i, j)$ such that $v_{i}(t(i, j))-v_{i}(t(i, j)-1) \geq c(i, j)$ if it exists. If it does not exist (i.e. $v_{i}(l)-v_{i}(l-1)<c(i, j)$ for all $\left.l=1,2, \ldots\right)$, we set $t(i, j)=0$.

Definition 5. For all $i, j \in N$,

$$
t(i, j)=\max \left\{\left\{l \in \mathbb{N} \mid v_{i}(l)-v_{i}(l-1) \geq c(i, j)\right\} ; 0\right\} .
$$

The existence of $t(i, j)$ is guaranteed by assumption $b$. For our example, we have $t(1,3)=1$.

Given the first and second step above, we can form, for each pair of agents $(i, j)$ $\left(i \in N_{t}, j \in N\right)$ the pair $\left(r\left(i, j, g_{t}\right), t(i, j)\right)$. These pairs are shown in the first four columns of table 2 below.

\begin{tabular}{|c||cccc|c|c|c|}
\hline \multicolumn{7}{|c}{} & \multicolumn{7}{c|}{$\left(r\left(i, j, g_{0}\right), t(i, j)\right)$} & $\Lambda_{i}\left(g_{0}\right)$ & $\rho_{i}\left(g_{0}\right)$ & $\rho_{i}\left(g_{0}\right)-\theta(i)$ \\
& 1 & 2 & 3 & 4 & & & \\
\hline 1 & $/$ & $\mathbf{( 2 , 2 )}$ & $(3,1)$ & $\mathbf{( 1 , 3 )}$ & $\{4,2\}$ & 44 & 34 \\
2 & $(3,2)$ & $/$ & $\mathbf{( 2 , 2 )}$ & $\mathbf{( 1 , 4 )}$ & $\{4,3\}$ & 38 & 33 \\
3 & $(3,1)$ & $(2,1)$ & $/$ & $\mathbf{( 1 , 2 )}$ & $\{4\}$ & 47 & 27 \\
4 & $\mathbf{( 2 , 3 )}$ & $\mathbf{( 1 , 3 )}$ & $(3,2)$ & $/$ & $\{2,1\}$ & 13 & $\mathbf{- 2}$ \\
\hline
\end{tabular}

Table 2: Summary table for $\mathrm{t}=0$

In a third step, we look for all pairs of agents $(i, j)$ with $i \in N_{t}$ and $j \in N$ with $i j \in g_{t}$ for which $r\left(i, j, g_{t}\right) \leq t(i, j)$. These are indicated in bold in the first four columns of table 2. The set of all these agents $j \in N$ will be equal to the set of 'acceptable' agents for $i$ in network $g_{t}$. We collect these in the set $\Lambda_{i}\left(g_{t}\right)$. This set of acceptable agents for agent $i$ can be interpreted as the best response for agent $i$ in the network $g_{t}$. In particular, the set $\Lambda_{i}\left(g_{t}\right)$ gives the agents that are linked to $i$ in network $g_{t}$ and with whom $i$ does not want to cut his link. 
Definition 6. For all $i \in N$ and $g \in \Lambda_{n}$,

$$
\Lambda_{i}(g)=\left\{j \in N \mid i j \in g_{t} \text { and } r\left(i, j, g_{t}\right) \leq t(i, j)\right\} .
$$

For the example, both agents 2 and 4 are acceptable for agent 1 in network $g_{0}$. Hence, $\Lambda_{1}\left(g_{0}\right)=\{4,2\}$. Observe that it is possible that $\Lambda_{i}(g)$ is empty for some $i$ and $g$. For our example, the set of acceptable agents is given in table 2 (second column) for round 0 .

Given that we have constructed the best responses for each agent $i$ in $N_{t}$. What is left to determine now is the set of agents $A_{t}$ who will change their strategy to their best responses strategy, i.e. who will be the agents in $N_{t}$ that will effectively delete all links $i j \in g_{t}$ for which $j \notin \Lambda_{i}\left(g_{t}\right)$ and keeping all other links of $i$ in $g_{t}$.

Therefore, we consider in the fourth step, for each $i \in N_{t}$ and each $j \in \Lambda_{i}\left(g_{t}\right)$, the cost $c(i, j)$ and we retain the highest value. We call this the critical cost for $i$ in $g_{t}$ and we denote it by $\rho_{i}\left(g_{t}\right)$. If $\Lambda_{i}\left(g_{t}\right)$ is empty, we set $\rho_{i}\left(g_{t}\right)=0$. For our example, we have that $c(1,2)>c(1,4)$, hence $c(1,2)=\rho_{1}\left(g_{0}\right)=44$ is the critical cost for agent 1 in $g_{0}$. From the discussion above, we see that agent $i$, in network $g$, would like to cut all links with agents $j$ for which $i j \in g$ and $c(i, j)>\rho_{i}(g)$.

Definition 7. For all $i, j \in N$ and all $g \in \mathcal{G}_{n}$, the critical cost for $i$ in $g$ is given by,

$$
\rho_{i}(g)=\max \left\{\left\{c(i, j) \mid j \in \Lambda_{i}(g)\right\}\right\} \text {, if } \Lambda_{i}(g) \neq \emptyset \text { and } \rho_{i}(g)=0 \text { otherwise. }
$$

Now, we compute for all agents $i \in N_{t}$ the value $\rho_{i}\left(g_{t}\right)-\theta(i)$ and we identify the collection of agents $i \in N_{t}$ for which $\rho_{i}\left(g_{t}\right)-\theta(i)$ is smallest. This collection determines $A_{t}$. For our numerical example, we obtain $A_{0}=\{4\}$.

The intuition behind the particular choice of $A_{t}$ is the following. We know that any agent $i \in N_{t}$ would like to cut all links $i j \in g_{t}$ for which $c(i, j)>\rho_{i}\left(g_{t}\right)$ (or equivalently, $j \notin \Lambda_{i}\left(g_{t}\right)$ ) and keep all links with $c(i, j) \leq \rho_{i}\left(g_{t}\right)$ (or equivalently $j \in \Lambda_{i}\left(g_{t}\right)$ ). Now consider an agent $i \in A_{t}$ and let $c(i, j) \leq \rho_{i}\left(g_{t}\right)$. Then,

$$
\begin{aligned}
c(j, i) & =d(i, j)-\theta(i) \\
& =c(i, j)-\theta(i)+\theta(j) \\
& \leq \rho_{i}\left(g_{t}\right)-\theta(i)+\theta(j) \\
& \leq \rho_{j}\left(g_{t}\right) .
\end{aligned}
$$

The first and second equality follows from the definition of $c(j, i)$ and $c(i, j)$. The first inequality follows from the fact that $c(i, j) \leq \rho_{i}\left(g_{t}\right)$ while the last inequality follows from the definition of $A_{t}$. As such, we see that $c(j, i) \leq \rho_{j}\left(g_{t}\right)$ or equivalently $i \in \Lambda_{j}\left(g_{t}\right)$, i.e. $i$ is an acceptable agent for $j$ in $g_{t}$. As such, $j$ will not have an incentive to cut his link with $i$ in network $g_{t}$.

We are now ready to update the algorithm to the next step. To construct $g_{t+1}$ from $g_{t}$ we take the collection of agents obtained from the previous step, $A_{t}$, and we let them cut the links with all agents $j$ not in $\Lambda_{i}\left(g_{t}\right)$. Considering our particular example, we have 
that $A_{0}=\{4\}$ and $\Lambda_{4}\left(g_{0}\right)=\{2,1\}$, hence, $g_{1}=g_{0} /\{\{3,4\}\}$. Next, we construct the set $N_{t+1}=N_{t}-A_{t}$, giving $N_{1}=\{1,2,3\}$. Finally, we go back to step 1 of the next iteration.

For our example, we see that in the next iteration, $A_{1}=\{2,3\}$ and that $g_{2}$ is formed by deleting the link between 1 and 2 and between 1 and 3 (see table 3 ). Note that since agent 4 has deleted his link with agent 3 , agent 2 became a member of the set of acceptable agents of agent $3, \Lambda_{3}\left(g_{1}\right)$.

\begin{tabular}{|c||cccc|c|c|c|}
\hline \multicolumn{1}{|c||}{} & \multicolumn{9}{c|}{$\left(s\left(i, j, g_{1}\right), k(i, j)\right)$} & $\Lambda_{i}\left(g_{1}\right)$ & $\rho_{i}\left(g_{1}\right)$ & $\rho_{i}\left(g_{1}\right)-\theta(i)$ \\
& 1 & 2 & 3 & 4 & & & \\
\hline 1 & $/$ & $\mathbf{( 2 , 2 )}$ & $(3,1)$ & $\mathbf{( 1 , 3 )}$ & $\{4,2\}$ & 44 & 34 \\
2 & $(3,2)$ & $/$ & $(\mathbf{2 , 2})$ & $\mathbf{( 1 , 4 )}$ & $\{4,3\}$ & 38 & 33 \\
3 & $(2,1)$ & $\mathbf{( 1 , 1 )}$ & $/$ & $/$ & $\{2\}$ & 53 & 33 \\
\hline
\end{tabular}

Table 3: Summary table for $\mathrm{t}=1$

\begin{tabular}{|c|c|c|c|c|}
\hline & $\left(s\left(i, j, g_{2}\right), k(i, j)\right)$ & $\Lambda_{i}\left(g_{2}\right)$ & $\rho_{i}\left(g_{2}\right)$ & $\rho_{i}\left(g_{2}\right)-\theta(i)$ \\
\hline & $\begin{array}{llll}1 & 2 & 3 & 4\end{array}$ & & & \\
\hline 1 & $/ / /(1,3)$ & $\left\{x_{4}\right\}$ & 8 & -2 \\
\hline
\end{tabular}

Table 4: Summary table for $\mathrm{t}=2$

Finally, we end with $N_{2}=\{1\}$ in round 2, see table 4 . No link is deleted in round 2 . This is a general feature of our algorithm: any link that is not deleted in a certain iteration $t$ will not be deleted further in the algorithm (see lemma 2 in the appendix). As such, we may end the algorithm as soon as $N_{t}$ is a singleton. We see that $g_{3}$ is the final network in the algorithm because $N_{3}=\emptyset$. We obtain $g_{3}=\{\{1,4\},\{2,3\},\{2,4\}\}$. The full algorithm is summarized in figure 1 .

The following theorem shows that a strong pairwise stable network always exists, in the form of the output of figure 1 , and that it is always unique. The proof can be found in the appendix.

Theorem 1. The set of strong pairwise stable networks $\Pi_{s p s}\left(\left\{\pi_{i}\right\}_{i \leq n}\right)$ is a singleton. The unique element of $\Pi_{s p s}\left(\left\{\pi_{i}\right\}_{i \leq n}\right), g_{s p s}$ is reproduced by the output of the algorithm in figure 1 .

Allowing for the simultaneous formation and cutting of a link, as presented in the definition of a strong pairwise stable network, is essential for uniqueness of the stable network. 
I. Initiate $t=0, N_{0}=N$ and let $\left(s_{1}^{0}, \ldots, s_{n}^{0}\right)$ be such that $g_{0}=g_{N}=g\left(s_{1}^{0}, \ldots, s_{n}^{0}\right)$.

1. Let $A_{t}=\left\{i \in N_{t} \mid \forall j \in N_{t}: \rho_{i}\left(g_{t}\right)-\theta(i) \leq \rho_{j}\left(g_{t}\right)-\theta(j)\right\}$

2. For all $i \in A_{t}$, define $s_{i}^{t+1}$ by:

$$
j \in s_{i}^{t+1} \text { if and only if } c(i, j) \leq \rho_{i}\left(g^{t}\right) .
$$

For all $j \notin A_{t}$ define $s_{j}^{t+1}=s_{j}^{t}$.

3. Set $N_{t+1}=N_{t}-A_{t}$ and $g^{t+1}=g\left(s_{1}^{t+1}, \ldots, s_{n}^{t+1}\right)$.

4. If $N_{t+1}=\emptyset$ go to step II, else, increase $t$ by one $(t:=t+1)$ and go to step I- 1 .

II. Set $g_{A}=g\left(s_{1}^{t+1}, \ldots, s_{n}^{t+1}\right)$ and end the algorithm.

Figure 1: Stable network algorithm.

Indeed, it is possible that there are multiple pairwise stable networks (see definition 3). To see this, consider an example with three agents $i, j$ and $k$ and assume that both $j$ and $k$ are willing to link with $i$ but not with each other. Further assume that $i$ would benefit from linking to either $j$ and $k$, but not to both. This happens if, for example,

$$
\begin{aligned}
& c(j, i)<v_{j}(1)-v_{j}(0)<c(j, k), \quad c(k, i)<v_{k}(1)-v_{k}(0)<c(k, j), \\
& v_{i}(1)-v_{i}(0)>c(i, k), c(i, j) \quad \text { and } \quad v_{i}(2)-v_{i}(1)<c(i, k), c(i, j) \text {. }
\end{aligned}
$$

Assume that $c(i, j)<c(i, k)$ and let $g=\{i k\}$. Since $i$ and $k$ have no other links, neither of them will have an incentive to cut the link $i k$. Agent $j$ may propose to form a link with $i$. In the pairwise stability framework, where $i$ cannot cut and form a link at the same time, $i$ will decline $j$ 's proposal although $i$ would like to cut his link with $k$ and link with $j$ because this would give him a positive net benefit of $c(i, k)-c(i, j)$. In this setting, the concept of pairwise stability does not lead to a unique stable network as both networks with $g=\{i j\}$ and $g=\{i k\}$ are pairwise stable. On the other hand, if we impose the strong pairwise stability concept, we see that, in this case, $i$ will cut his link with $k$ and accept the proposal to link with $j$ at the same time leaving only $g=\{i j\}$ to be strong pairwise stable.

As for the strongly stable network, we show in the following proposition that the unique strong pairwise stable network is also strongly stable. This implies that if we would impose no restrictions on the actions available to the agents and the size of the deviating coalition the strong pairwise stable network would still be stable.

Proposition 1. The unique strong pairwise stable network $g_{\text {sps }}$ is strongly stable:

$$
\Pi_{s s}\left(\left\{\pi_{i}\right\}_{i \leq n}\right)=\Pi_{s p s}\left(\left\{\pi_{i}\right\}_{i \leq n}\right)
$$


The uniqueness of the weakly and strongly stable network hinges on the following property of the payoff structure: if $c(i, j)<c(i, k)$ and $c(j, k)<c(j, i)$, then $c(k, j)<c(k, i)$ - if $i$ prefers $j$ to $k$ and $j$ prefers $k$ to $i$, then $k$ will prefer $j$ to $i$. Hence the preferences of $k$ are such that the objective of two players ( $\mathrm{j}$ and $\mathrm{k}$ ) are aligned. This rules out any cycles in the preference structure and uniqueness ensues.

We finalize this section by giving an alternative cost structure for which above existence and uniqueness result remains to hold. In the basic model, we assumed that $c(i, j)$ could be written as the difference between a symmetric distance function $d(i, j)$ and an agent specific quality function $\theta(j)$. On the other hand, we could also specify $c(i, j)$ as the ratio of a symmetric distance function $d(i, j)$ and an agent specific (non-zero) quality function $\theta(j): N \rightarrow \mathbb{R}_{++}:$

$$
c(i, j)=\frac{d(i, j)}{\theta(j)} .
$$

All the results of this section are valid for this cost function with a minor adjustment in the definition of the set, $A_{t}$, which is used in step I-1 in the algorithm of figure 1 , which changes to,

$$
A_{t} \in\left\{i \in N_{t} \mid \forall j \in N_{t}: \frac{\rho_{i}\left(g_{t}\right)}{\theta(i)} \leq \frac{\rho_{j}\left(g_{t}\right)}{\theta(j)}\right\} .
$$

\section{Empirical Illustration}

Using data from a risk sharing network in Nyakatoke, Tanzania, we verify whether our model is able to predict the existence of such risk sharing links and whether it can capture the features of risk sharing networks observed in real life. Informal risk sharing networks - networks where linking partners have an informal mutual agreement to help each other when one partner incurs a negative shock - are particularly well suited examples of the payoff structure under consideration.

In the literature on informal risk-sharing networks, for example, De Weerdt (2002) finds that kinship, geographical proximity, clan membership and religious affiliation are strong determinants in the formation of risk-sharing networks. This result is corroborated by the results of Fafchamps and Gubert (2007) who find that geographic proximity, kith and kin relationships are strong determinants of link formation within an informal insurance network in the rural Philippines. Hence we can think of the pairwise component $d(i, j)$ as a notion of social and geographical distance. Households who live close by or know each other well through friendship or kinship ties can link to each other at lower costs.

In addition, empirical studies on risk-sharing networks reveal that some agents are more desirable linking partners than others because they have more wealth or status. For example, Comola (2008) and De Weerdt (2002), find that the probability of being linked to a household rises with the wealth of this household, that the rich have a denser network than the poor and that a dyad with a rich person in it has more chance of being linked. Hence, households are more willing to link to a household of higher wealth which is incorporated in our model by the agent-specific component $\theta($.$) .$ 
We keep this illustration as simple as possible, using the geographical distance between two households as a proxy for our distance measure $d(i, j)$, while the value of livestock owned by a household serves as a proxy for our quality measure $\theta(i)$. Various empirical studies ${ }^{8}$ on risk sharing networks identify geographical proximity as one of the important determinants of risk sharing links. For the case of livestock, Comola (2008) argues that livestock is a real wealth dimension, more so than land ownership.

At the time of the survey in 2000, Nyakatoke consisted of 119 households, 116 for which data are fully available. All households were asked the following question: Can you give a list of people [...], who you can personally rely on for help and/or that can rely on you for help in cash, kind or labour? The 116 households mentioned a total of 960 intra-village network partners, which amounts to 480 risk sharing links. However, the degree (i.e. the number of network partners) is unevenly distributed, ranging from 1 to 32 partners. Furthermore, the Nyakatoke risk sharing network has features corresponding to other empirical regularities of large social networks, as noted by Comola (2008): there is only one component which has an average path length of 2.5 steps, clustering is higher than would be expected if the formation process would be totally random and clustering tends to be negatively correlated with degree. ${ }^{9}$ The key characteristics of the Nyakatoke network are shown in the first column of table 6 below.

As a measure of predictive power we look at the fraction of existing links in the Nyakatoke network that also exist in the stable network produced by the algorithm, as well as the fraction of non-existing links in the Nyakatoke network that do not exist in the stable network. In order to get a sense of how accurate the predictions based on the model are, we compare our results with predictions based on using fitted link probabilities from a logit regression model using geographical distance and/or livestock values. Since both predictions rely on the same set of information - geographical distance and livestock value we are able to filter out the contribution of our network formation model by comparing the two prediction fractions. We fully realize we use very crude measures of distance and quality and in this respect this exercise should not be viewed as an empirical application but rather as a way of showing that the network formation process presented in this paper might have some validity when it comes to predicting the existence of links.

First, we focus on distances only, simply inputting geographical distances between any pair while assuming $\theta(i)=0$ for all $i \in N$. The payoff function is of the form

$$
\pi_{i}(g)=\alpha * n_{i}(g)^{2 / 3}-\sum_{j \in L_{i}(g)} d(i, j)
$$

with the scale parameter $\alpha$ chosen such that the total number of links in the network is as close to 480 (the total number of links in the real-life network) as possible. For this exercise, $\alpha=0,454$, with a total number of 479 links. The exponent for the benefit function is fixed at $\frac{2}{3}$. Simulations with other values within the range of $\left[\frac{2}{5}, \frac{4}{5}\right]$ yielded similar results.

\footnotetext{
${ }^{8}$ See Fafchamps and Lund (2003), Fafchamps and Gubert (2007), De Weerdt (2002) and Comola (2008).

${ }^{9}$ Another empirical regularity is a positive correlation in degree of connected households for which we could not find evidence in the Nyakatoke risk sharing network.
} 


\begin{tabular}{|r||c|c|}
\hline Fraction of Correct predictions & Linked & Unlinked \\
\hline Model (only distance) & 0,313 & 0,947 \\
Fitted Probability (only distance) & {$[0,088-0,133]$} & {$[0,926-0,936]$} \\
Model (distance and quality) & 0,333 & 0,948 \\
Fitted Probability (distance and quality) & {$[0,098-0,146]$} & {$[0,927-0,937]$} \\
\hline
\end{tabular}

Table 5: Prediction results

The prediction results are given in the first row of table 5. Keeping in mind that our handling of the data is rather raw, the results are mixed. Somewhat less than one third of all existing links is predicted in our model. Earlier simulations - not shown here show that if we randomly select 480 pairs to be linked, we get a prediction rate for linked pairs between 3 and $13 \%$ and between 92,5 and $93,2 \%$ for unlinked pairs. Hence, we get prediction rates well above what we would expect from a non-informative model.

In order to filter out the contribution of our model to predicting the Nyakatoke network, we compare the results from the first row of table 5 with the prediction results from the fitted probability model as shown in the second row. In this model, we fitted the following logit regression with standard errors in parentheses:

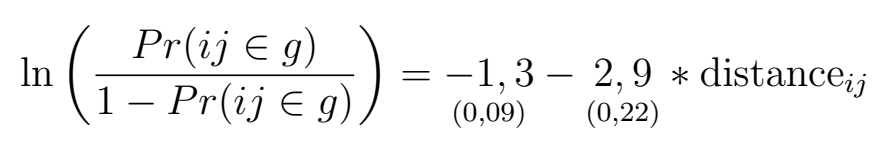

Then, networks are simulated where the probability that a link exists between a pair is equal to the fitted probability from the logit regression. The numbers shown in the table are the [5-95] percentiles, obtained over 100000 simulations. The prediction rates for our model are above these intervals, indicating that the network formation process as described in this paper is relevant to explaining the existence or absence of certain links in the Nyakatoke risk sharing network.

The simulation with the quality measure is a bit more complex. The quality measure $\theta(i)$ is the value of livestock of $i$. First, since the livestock value is of a different magnitude than the geographical distance measure, ${ }^{10}$ we scale it down using the ratio of coefficients from the following logit regression:

$$
\ln \left(\frac{\operatorname{Pr}(i j \in g)}{1-\operatorname{Pr}(i j \in g)}\right)=\underset{(0,09)}{-1,4}-\underset{(0,22)}{3} * \text { distance }_{i j}+\underset{\left(1,3 * 10^{-7}\right)}{1} * 10^{-6} * \sum_{k=i, j} \text { livestock }_{k}
$$

which turns out to be $\frac{3}{1000000}$. Second, adding quality adds a weighting parameter $\beta$ to

\footnotetext{
${ }^{10}$ Distance is expressed in kilometers with values ranging from close to 0 to 1,8 , while livestock can take on values to over 1,5 million.
} 
the net benefit function:

$$
\pi_{i}(g)=\alpha * n_{i}(g)^{2 / 3}-\sum_{j \in L_{i}(g)}((\beta * d(i, j)-(1-\beta) * \theta(j)) .
$$

For different values of $\beta$ - between 0 and 1 with steps of 0,02 - we calibrate $\alpha$ such that the total number of links is approximately 480. The third row of table 5 gives the prediction results for the $\{\beta, \alpha\}$-combination which yielded the best prediction results, $\{\beta=0,48, \alpha=2,49\}$. Again, comparing these results with the results from the fitted probability model with distance and quality as shown in the fourth row indicates that our model can help explain the existence of links in the Nyakatoke network, although prediction fractions do not improve markedly when adding quality.

\begin{tabular}{|c|c|c|c|}
\hline & Nyakatoke & Model (distance) & Model (distance and quality) \\
\hline [Min Median Max] & {$\left[\begin{array}{lll}1 & 7 & 32\end{array}\right]$} & {$\left[\begin{array}{lll}1 & 8 & 16\end{array}\right]$} & {$\left[\begin{array}{lll}3 & 8 & 10\end{array}\right]$} \\
\hline Char. Path length & 2,54 & 4,23 & 3,64 \\
\hline Clustering & 0,19 & 0,62 & 0,52 \\
\hline Corr. Clust. - Degree & $-0,21$ & $-0,043$ & $-0,39$ \\
\hline Corr. Degree linked agents & 0,04 & 0,70 & 0,49 \\
\hline
\end{tabular}

Table 6: Key statistics of networks: The clustering coefficient used is the overall clustering coefficient (see ?).

As far as the features of the simulated networks, shown in table 6 , are concerned, we can say that they match at some points the features of networks observed in real life high degree of clustering, negative correlation between clustering and degree and positive correlation in degree of connected agents. At other points - the uneven distribution of degree as well as the low characteristic path length — the model fails to replicate, which can be attributed to the usage of the raw distance and quality measures. For example, geographical distance is a measure which is a metric, satisfying triangle inequality ${ }^{11}$, hence producing high characteristic path lengths. If we would introduce more accurate social distance measures, which would not necessarily satisfy triangle inequality, characteristic path length may be lower. The inaccuracy of the data may also explain the fact that we did not obtain an uneven degree distribution. Additionally, we assumed identical benefit functions for every household. Introducing some heterogeneity on the benefit side may lead to a more uneven degree distribution.

\section{Concluding remarks}

In an endogenous network formation setting where only the direct network matters for utility, this paper introduced general forms of heterogeneity on the cost side. The costs are

${ }^{11} d(i, j)+d(j, k) \geq d(i, k)$ 
characterized by the distance between agents and individual quality. The only restrictions we impose is that the distance is symmetric, that each agent has a perfect ordering of linking costs without indifference and that agents are never indifferent between forming and cutting a link. Using an algorithm we were able to identify the unique strong pairwise stable network. agents will try to link with agents with the lowest linking costs so as to maximize the net gains from the direct network. Not everyone will succeed in doing this however, as some desirable network partners may refuse to form a link with them.

The algorithm provides an elegant way to determine the emerging unique stable network. Different structures will give rise to different configurations and hence, using the framework, it might become possible to relate differences in features of networks to differences in underlying cost structure.

The uniqueness result of the stable network can be attributed to two factors. Firstly there are the assumptions made regarding the cost function - perfect preference ordering and symmetric distance function — as well as on the actions available to the agents the fact that agents can simultaneously cut and form a link. Relaxing the assumptions would invalidate the uniqueness result by including some history dependence in the model. However, the basic configuration of the emerging stable networks will be little affected by this relaxation, so that we can consider this kind of randomness as irrelevant to the model.

A second set of factors which leads to uniqueness of the stable network are the specific structure of the link-costs — with a pairwise and a agent-specific component — which precludes preference cycles and the assumptions made with respect to the utility function

- absolute friction and concavity. Hence, relaxing the assumptions with respect to the link-costs even further as well as imposing more intermediate forms of friction may be an important direction for future research.

\section{References}

Bala, V., Goyal, S., 2000. A Non-cooperative Model of Network Formation. Econometrica 68, 205-228.

Belleflamme, P., Bloch, F., 2004. Market Sharing Agreements and Collusive Networks. International Economic Review 45, 387-411.

Carayol, N., Roux, P., 2006. A Strategic Model of Complex Network Formation. BETA working paper 2:14.

Comola, M., 2008. The Network Structure of Informal Arrangements : Evidence from Rural Tanzania. Laboratoire d'Economie Appliquee, INRA, Research Unit Working Papers, 0708.

De Weerdt, J., 2002. Risk-Sharing and Endogenous Network Formation. World Institute for Development Economic Research (UNU-WIDER), Working Paper. 
De Weerdt, J., Dercon, S., 2006. Risk-Sharing Networks and Insurance against Illness. Journal of Development Economics 81, 337-356.

Deroian, F., 2003. Farsighted Strategies in the Formation of a Communication Network. Economics Letters 80, 343-349.

Dutta, B., Ghosal, S., Ray, D., 2005. Farsighted Network Formation. Journal of Economic Theory 122, 143-164.

Dutta, B., Jackson, M. O., 2000. The Stability and Efficiency of Directed Communication Networks. Review of Economic Design 5, 251-272.

Dutta, B., Mutuswami, S., 1997. Stable Networks. Journal of Economic Theory 76, 322344.

Dutta, B., Tijs, S., van den Nouweland, A., 1998. Link Formation in Cooperative Situations. International Journal of Game Theory 27, 245-256.

Fafchamps, M., Gubert, F., 2007. The Formation of Risk Sharing Networks. Journal of Development Economics 83, 326-350.

Fafchamps, M., Lund, S., 2003. Risk-Sharing Networks in Rural Phillipines. Journal of Development Economics 71, 261-287.

Feri, F., 2007. Network Formation with Endogenous Decay. university of Innsbruck Working Paper in Economics and Statistics, 2007-14.

Galeotti, A., Goyal, S., Kamphorst, J., 2006. Network Formation with Heterogeneous Players. Games and Economic Behavior 54, 353-372.

Haller, H., Sarangi, S., 2005. Nash Networkds with Heterogeneous Links. Mathematical Social Sciences 50, 181-201.

Jackson, M., Rogers, B. W., 2005. The Economics of Small Worlds. Journal of the European Economic Association 3, 617-627.

Jackson, M., van den Nouweland, A., 2005. Strongly Stable Networks. Games and Economic Behavior 51, 420-444.

Jackson, M., Wolinsky, A., 1996. A Strategic Model of Social and Economic Networks. Journal of Economic Theory 71, 44-74.

Jackson, M. O., Watts, A., 2001. The Existence of Pairwise Stable Networks. Seoul Journal of Economics 14, 299-321.

Jackson, M. O., Watts, A., 2002. The Evolution of Social and Economic Networks. Journal of Economic Theory 106, 265-295. 
Johnson, C., Gilles, R., 2000. Spatial Social Networks. Review of Economic Design 5, 273-299.

Myerson, R. B., 1991. Game Theory: Analysis of Conflict. Harvard University Press, Cambridge.

Slikker, M., van den Nouweland, A., 2000. Network Formation Models with Cost for Establishing Links. Review of Economic Design 5, 333-362.

Slikker, M., van den Nouweland, A., 2001. A One-Stage Model of Link Formation and Payoff Division. Games and Economic Behavior 34, 153-175.

Watts, A., 2002. Non-Myopic Formation of Circle Networks. Economics Letters 74, 277282.

\section{A Proofs}

\section{A.1 Preliminary results}

Before proving theorem 1, we introduce some lemmata.

Lemma 1. If $i \in A_{t}, i j \in g_{t+1}$ and $s \geq t$, then $i j \in g_{s+1}$.

Proof. Assume that $i \in A_{t}$ and $i j \in g_{t+1}$. Further, let $j \in A_{p}$. If $p<t$, we have, by $g_{t+1} \subseteq g_{p+1}$, that $i j \in g_{p+1}$. As there is no iteration between $t$ and $s$ where $i j$ could be cut, we conclude that $i j \in g_{s+1}$

Therefore assume that $p \geq t$. Observe that it suffices to show that $c(j, i) \leq \rho_{j}\left(g_{p}\right)$ because this guarantees that $j$ will not cut the link $i j$ in iteration $p$ of our algorithm. From $i \in A_{t}$ and $i j \in g_{t+1}$, we conclude that,

$$
c(j, i)=c(i, j)-\theta(i)+\theta(j) \leq \rho_{i}\left(g_{t}\right)-\theta(i)+\theta(j) \leq \rho_{j}\left(g_{t}\right) .
$$

This implies,

$$
r\left(j, i, g_{t}\right) \leq t(j, i)
$$

and from $g_{p} \subseteq g_{t}$, we derive,

$$
r\left(j, i, g_{p}\right) \leq r\left(j, i, g_{t}\right) \leq t(j, i)
$$

Hence, $c(j, i) \leq \rho_{j}\left(g_{p}\right)$.

An important corollary of lemma 1 is that for all iterations $t$ of the algorithm and $i \in A_{t}$, if $i j \in g_{t+1}$, then $i j \in g_{A}$ (here $g_{A}$ is the output of the algorithm).

Lemma 2. If the network $g$ is strong pairwise stable and $g \subseteq g^{\prime}$, then for all ij $\in g^{\prime}$, if $c(i, j) \leq \rho_{i}\left(g^{\prime}\right)$ and $c(j, i) \leq \rho_{j}\left(g^{\prime}\right)$, then $i j \in g$. 
Proof. Assume that $c(i, j) \leq \rho_{i}\left(g^{\prime}\right)$ and $c(j, i) \leq \rho_{j}\left(g^{\prime}\right)$. If $i j \notin g$, then by strong pairwise stability of $g$ it follows that (using assumption e),

$$
v_{i}\left(n_{i}(g)+1\right)-v_{i}\left(n_{i}(g)\right)<c(i, j) \quad \text { or } \quad v_{j}\left(n_{j}(g)+1\right)-v_{j}\left(n_{j}(g)\right)<c(j, i) .
$$

Otherwise we would have that both $i$ and $j$ would benefit from creating the link $i j$. This shows that either $t(i, j)<n_{i}(g)+1$ or $t(j, i)<n_{j}(g)+1$. Assume without loss of generality that the first of the two holds. From $c(i, j) \leq \rho_{i}\left(g^{\prime}\right)$, it follows that

$$
r\left(i, j, g^{\prime}\right) \leq t(i, j) \leq n_{i}(g)
$$

As $g \subseteq g^{\prime}, i j \in g^{\prime}$ and $i j \notin g$, it follows that there must be an agent, $k$, which is linked to $i$ in $g$ and for which $c(i, k)>c(i, j)$. This shows that $i$ would benefit by cutting the link with $k$ and creating the link with $j . g$ is strong pairwise stable, hence, it must be that this is not beneficial for $j$, i.e.

$$
v_{j}\left(n_{j}(g)+1\right)-v_{j}\left(n_{j}(g)\right)<c(j, i) .
$$

This implies $t(j, i)<n_{j}(g)+1$. Parallel to the previous case, we conclude that there must be an agent $h$ such that $j h \in g$ and $c(j, h)>c(j, i)$.

Conclude that both $i$ and $j$ can benefit from linking with each other and cutting their link with respectively $k$ and $h$. Therefore, $g$ cannot be strong pairwise stable.

\section{A.2 Proof of theorem 1}

We only show uniqueness. The property that the output of the algorithm $g_{A}$ is strong pairwise stable follows from the proof of proposition 1 as every strongly stable network is also strong pairwise stable.

Therefore, assume that there exists a strong pairwise stable network $g_{\text {sps }}$. We need to show that $g_{A}=g_{\text {sps }}$. We work by induction on the iterations in the algorithm. In particular we show that for all $t=0,1, \ldots, T-1$,

1. if $i \in A_{t}$ and $i j \in g_{t+1}$, then $i j \in g_{s p s}$,

2. $g_{s p s} \subseteq g_{t+1}$.

If these two conditions hold, then from lemma 1 it follows that $g_{s p s}=g_{T}=g_{A}$.

First of all, observe that $g_{\text {sps }} \subseteq g_{0}=g_{N}$. Consider the case $t=0$. Now, let $i \in A_{0}$ and $i j \in g_{1}$. It follows that $c(i, j) \leq \rho_{i}\left(g_{0}\right)$. As such,

$$
c(j, i)=c(i, j)-\theta(i)+\theta(j) \leq \rho_{i}\left(g_{0}\right)-\theta(i)+\theta(j) \leq \rho_{j}\left(g_{0}\right) .
$$

It follows, from lemma 2 that $i j \in g_{\text {sps }}$. In addition, consider $i \in A_{0}$ and $i h \notin g_{1}$. It follows that $c(i, h)>\rho_{i}\left(g_{0}\right)$ or equivalently

$$
v_{i}\left(n_{i}\left(g_{1}\right)\right)-v_{i}\left(n_{i}\left(g_{1}\right)-1\right)<c(i, h) .
$$


Assume that $i h \in g_{s p s}$. Since all $i j \in g_{t+1}$ are part of $g_{s p s}$, it must be that $n_{i}\left(g_{1}\right)<n_{i}\left(g_{s p s}\right)$. Then,

$$
v_{i}\left(n_{i}\left(g_{s p s}\right)-v_{i}\left(n_{i}\left(g_{s p s}\right)-1\right)<c(i, h) .\right.
$$

As such, $i$ will benefit from cutting his link with $h$ in $g_{s p s}$. Hence, $g_{s p s}$ cannot be a stable network. We have that $i h \notin g_{s p s}$ and $g_{s p s} \subseteq g_{1}$.

Assume that the induction holds for all iterations up to $t-1$ and consider iteration $t$. Let $i \in A_{t}$ and $i j \in g_{t+1}$, then

$$
c(j, i)=c(i, j)-\theta(i)+\theta(j) \leq \rho_{i}\left(g_{t}\right)-\theta(i)+\theta(j) \leq \rho_{j}\left(g_{t}\right) .
$$

Again, using lemma 2, it follows that $i j \in g_{\text {sps. }}$. This shows the first requirement. We still need to show the second requirement. Towards this end, assume on the contrary that for some $i \in A_{t}, n_{i}\left(g_{t}\right) \geq n_{i}\left(g_{s p s}\right)>n_{i}\left(g_{t+1}\right)$. From lemma 1 one can easily deduce that for all $i \in A_{t}, \rho_{i}\left(g_{t}\right)=\rho_{i}\left(g_{t+1}\right)$. Hence for $i j \in g_{s p s}, g_{t}$ and $i j \notin g_{t+1}$,

$$
c(i, j)>\rho_{i}\left(g_{t+1}\right) \quad \text { or equivalently } \quad v_{i}\left(n_{i}\left(g_{t+1}\right)\right)-v_{i}\left(n_{i}\left(g_{t+1}\right)-1\right)<c(i, j) .
$$

From $n_{i}\left(g_{s p s}\right)>n_{i}\left(g_{t+1}\right)$, we deduce that,

$$
v_{i}\left(n_{i}\left(g_{s p s}\right)-v_{i}\left(n_{i}\left(g_{s p s}\right)-1\right)<c(i, j) .\right.
$$

As such, $i$ will benefit from cutting his link with $j$ in $g_{s p s}$ and $g_{s p s}$ cannot be a stable network. Now, we have that $g_{s p s} \subseteq g_{t}, n_{i}\left(g_{s p s}\right) \leq n_{i}\left(g_{t+1}\right)$ for all $i \in A_{t}$ and $i j \in\left(g_{t}-g_{t+1}\right)$ only if $i$ or $j \in A_{t}$. Conclude that $g_{s p s} \subseteq g_{t+1}$.

\section{A.3 Proof of proposition 1}

Take any $C \subseteq N$ and let $g_{A}$ be the output of the algorithm. We prove that there will always be at least one agent $i \in C$ who loses by any rearrangement of the linking pattern among the members of $C$, under the condition that links are formed under mutual consent. Consider an agent, $i$, defined by:

$$
i \in\left\{j \in C \mid j \in A_{t} \text { and } \forall k \in C \text { if } k \in A_{p} \text {, then } t \leq p\right\},
$$

Agent $i$ is a the member of $C$ who, in our algorithm, cuts links first.

Let $g_{A}$ be the output of the algorithm and let $g^{\prime}$ be a network where only links within $C$ are rearranged and perhaps links with at least one agent in the coalition are cut. Further, assume on the contrary, that all agents within $C$ benefit from changing from $g_{A}$ to $g^{\prime}$. Let $A=L_{i}\left(g_{A}\right) \backslash L_{i}\left(g^{\prime}\right)$ and $B=L_{i}\left(g^{\prime}\right) \backslash L_{i}\left(g_{A}\right)$. $A$ is the set of agents who are linked to $i$ in network $g_{A}$ but not in $g^{\prime}$ and $B$ is the set of agents who are linked to $i$ in $g^{\prime}$ but not in $g_{A}$. We assume that at least one of the sets $A$ or $B$ is nonempty. Otherwise, $i$ is indifferent between network $g_{A}$ and $g^{\prime}$. In that case, we may exclude $i$ from the set $C$ and consider the smaller set $C-\{i\}$. Now, for all $j \in N$, if $i j \in g_{t+1}$, then it follows (from lemma 1) that $i j \in g_{A}$. This implies that whenever $j \in A$ then $c(i, j) \leq \rho_{i}\left(g_{t}\right)$. On the other 
hand, because $i$ is the first agent to choose in the algorithm, it must be that for all $j \in B$, $c(i, j)>\rho_{i}\left(g_{t}\right)$. Conclude that for all $j \in A$ and $k \in B: c(i, j)<c(i, k)$. We have that:

$$
\pi_{i}\left(g_{A}\right)-\pi_{i}\left(g^{\prime}\right)=v_{i}\left(n_{i}\left(g_{A}\right)\right)-v_{i}\left(n_{i}\left(g^{\prime}\right)\right)-\sum_{j \in A} c(i, j)+\sum_{j \in B} c(i, j) .
$$

If $|A|=|B|$, the proof follows from the facts that $c(i, j)<c(i, k)$ for all $j \in A$ and $k \in B$ and $n_{i}\left(g_{A}\right)=n_{i}\left(g^{\prime}\right)$. We distinguish two other cases.

(1) If $|B|>|A|$, we can write:

$$
\pi_{i}\left(g_{A}\right)-\pi_{i}\left(g^{\prime}\right)=\sum_{k=0}^{|B|-|A|-1}\left(v_{i}\left(n_{i}\left(g_{A}\right)+k\right)-v_{i}\left(n_{i}\left(g_{A}\right)+k+1\right)\right)-\sum_{j \in A} c(i, j)+\sum_{j \in B} c(i, j) .
$$

Now, select $|A|$ agents from $B$ and call the remaining set $B^{\prime}$, then:

$$
\pi_{i}\left(g_{A}\right)-\pi_{i}\left(g^{\prime}\right)>\sum_{k=0}^{|B|-|A|-1}\left(v_{i}\left(n_{i}\left(g_{A}\right)+k\right)-v_{i}\left(n_{\iota}\left(g_{A}\right)+k+1\right)\right)+\sum_{j \in B^{\prime}} c(i, j) .
$$

For all $k \geq 0$ and $j \in B$, it is also the case that:

$$
v_{i}\left(n_{i}\left(g_{A}\right)+k+1\right)-v_{i}\left(n_{i}\left(g_{A}\right)+k\right)<c(i, j) .
$$

Hence,

$$
\pi_{i}\left(g_{A}\right)-\pi_{i}\left(g^{\prime}\right)>-\sum_{j \in B^{\prime}} c(i, j)+\sum_{j \in B^{\prime}} c(i, j)=0 .
$$

(2) Now, consider the case where $|A|>|B|$. Then we can write:

$$
\pi_{i}\left(g_{A}\right)-\pi_{i}\left(g^{\prime}\right)=\sum_{k=0}^{|A|-|B|+1}\left(v_{i}\left(n_{i}\left(g_{A}\right)-k\right)-v_{i}\left(n_{i}\left(g_{A}\right)-k-1\right)\right)-\sum_{j \in A} c(i, j)+\sum_{j \in B} c(i, j) .
$$

Select $|B|$ agents from $A$ and call the remaining set $A^{\prime}$. This gives:

$$
\pi_{i}\left(g_{A}\right)-\pi_{i}\left(g^{\prime}\right)>\sum_{k=0}^{|A|-|B|-1}\left(v_{i}\left(n_{i}\left(g_{A}\right)-k\right)-v_{i}\left(n_{i}\left(g_{A}\right)-k-1\right)\right)-\sum_{j \in A^{\prime}} c(i, j) .
$$

For all $k \geq 0$ and $j \in A$, it is also the case that:

$$
v_{i}\left(n_{i}\left(g_{A}\right)-k\right)-v_{i}\left(n_{i}\left(g_{A}\right)-k-1\right)>c(i, j) .
$$

Hence,

$$
\pi_{i}\left(g_{A}\right)-\pi_{i}\left(g^{\prime}\right)>\sum_{j \in A^{\prime}} c(i, j)-\sum_{j \in A^{\prime}} c(i, j)=0 .
$$

Conclude that $i$ has higher payoffs in $g_{A}$ than in $g^{\prime}$ contradicting the assumption that $g_{A}$ is not strongly stable. 